\title{
AN ADIABATIC INVARIANT FOR NONLINEAR THERMOELASTIC RODS UNDER COMPRESSION*
}

BY

\author{
W. A. DAY \\ Hertford College, Oxford
}

Introduction. The most familiar instance of an adiabatic invariant occurs in connection with Lorentz's problem, i.e., the motion of a pendulum whose length changes slowly. In that problem the angle $\phi(t)$ which the pendulum makes with the downward vertical satisfies the differential equation

$$
L(t) \ddot{\phi}+g \phi=0,
$$

the variable coefficient $L(t)$ being the length, and $g$ the constant acceleration due to gravity. It is known that the energy is approximately proportional to the local frequency

or, in other words, that

$$
\frac{1}{2 \pi}\left(\frac{g}{L}\right)^{1 / 2}
$$

$$
L^{1 / 2} \dot{\phi}^{2}+g L^{-1 / 2} \phi^{2}
$$

is an adiabatic invariant; even though $L$ is presumed to change slowly, it may, nonetheless, undergo large changes over time intervals of large duration, and the same is true of the energy.

A mechanical clock, whose pendulum expands or contracts with seasonal variations of temperature, provides an example of what Lorentz had in mind and, accordingly, it is tempting to speculate that, for a thermoelastic body immersed in an environment whose temperature varies slowly, there may exist an adiabatic invariant which is constructed from, among other things, the energy of the body and its volume or its length.

My purpose is to point out that such an adiabatic invariant does exist for certain nonlinear thermoelastic rods which are perfect conductors of heat.

The methods employed here are similar to those of my papers [1,2], in which adiabatic invariants are constructed for some purely mechanical problems; the methods owe much in principle, if not in detail, to Littlewood's treatment of Lorentz's problem $[3,4]$.

Although thermodynamic concepts obtrude inevitably into the discussion, the adjective "adiabatic" will be used only in conjunction with "invariant"; at no point does it bear the meaning that is customary in thermodynamics proper.

${ }^{*}$ Received November 6, 1987.

(C)1989 Brown University 
The problem. We consider a straight rod undergoing longitudinal motion; one end of the rod is held fixed while the other is subjected to a constant compressional force of amount $p$. In order to simplify matters, we suppose the rod to possess a reference configuration in which its length and its mass density both take the value unity, and with respect to which the material response is homogeneous. A particle of the rod will be labelled by the point $x$, of the unit interval $[0,1]$, which it occupies when the rod is placed in the reference configuration; thus, $x$ is a material, or Lagrangian, coordinate.

The displacement $u(x, t)$ is required to be $C^{2}$ in the half-strip $[0,1] \times[0, \infty)$ of the $(x, t)$-plane, and to satisfy the inequality

$$
1+u_{x}>0
$$

in which $u_{x}=\partial u / \partial x$. The inequality ensures that, for each $t \geq 0$, the deformation $x \rightarrow x+u(x, t)$ preserves the strict order of particles on the line, i.e., $x+u(x, t)<$ $y+u(y, t)$ whenever $0 \leq x<y \leq 1$.

The mass density of the deformed rod is

$$
\rho=1 /\left(1+u_{x}\right)
$$

and its length is

$$
L=1+u(1, t)
$$

We note that

$$
0 \leq x+u(x, t) \leq L(t)
$$

a fact which will prove to be important.

The rod is immersed in an environment whose absolute temperature is $\theta(t)$. The supposition that the rod is a perfect conductor of heat means that its temperature is independent of $x$ and coincides with $\theta(t)$ everywhere.

In the absence of body forces, the equation of motion is

$$
\sigma_{x}=u_{t t},
$$

where the stress $\sigma$ is determined by the density and the temperature through a constitutive relation

$$
\sigma(x, t)=\hat{\sigma}(\rho(x, t), \theta(t)) .
$$

The conditions at the ends of the rod are

$$
u(0, t)=0, \quad \sigma(1, t)=-p .
$$

At a later stage, $p$ will be required to be sufficiently large and $\theta(t)$ will be required to be slowly-varying.

If we substitute for $\sigma$ and $\rho$ in terms of $u$ we see that $u$ is a solution of the nonlinear equation

and satisfies the boundary equations

$$
-\hat{\sigma}_{\rho}\left(\frac{1}{1+u_{x}}, \theta(t)\right) u_{x x}=\left(1+u_{x}\right)^{2} u_{t t},
$$

$$
u(0, t)=0, \quad \hat{\sigma}\left(\frac{1}{1+u_{x}(1, t)}, \theta(t)\right)=-p .
$$

The nonlinear partial differential equation is the counterpart, in the present context, of the linear ordinary differential equation which arises in Lorentz's problem. 
Restrictions on material response. The construction of an adiabatic invariant depends upon a severe restriction on the function $\hat{\sigma}$; it is supposed that $\hat{\sigma}$ has the form

or, equivalently,

$$
\hat{\sigma}(\rho, \theta)=-\rho^{2} T(\theta) S^{\prime}\left(\frac{\rho}{T(\theta)}\right)
$$

where

$$
\hat{\sigma}=-\rho^{2} \hat{\psi}_{\rho}
$$

$$
\hat{\psi}(\rho, \theta)=T(\theta)^{2} S(\tau), \quad \tau=\rho / T(\theta),
$$

$S^{\prime}(\tau)$ being the derivative of $S(\tau)$. The functions $T$ and $S$ are themselves restricted by requiring $T(\theta)$ to be $C^{2}$ and strictly positive for $\theta>0$, and requiring $S(\tau)$ to be $C^{1}$ and to satisfy the inequalities

$$
\tau S(\tau) \geq-a \quad \text { and } \quad \tau\left|S^{\prime}(\tau)\right| \leq b \quad \text { for } \quad \tau>0,
$$

in which $a$ and $b$ are positive constants.

It is a crucial feature of these hypotheses that they imply the identity

$$
\hat{\psi}_{\theta}=\frac{T^{\prime}(\theta)}{T(\theta)}\left(2 \hat{\psi}+\frac{\hat{\sigma}}{\rho}\right) .
$$

The hypotheses are satisfied if

$$
T(\theta)=(R \theta)^{1 / 2}, \quad S(\tau)=\log \tau, \quad a=\exp (-1), \quad b=1,
$$

where $R$ is a positive constant. In this case

$$
\hat{\sigma}=-R \rho \theta, \quad \hat{\psi}=R \theta \log \left(\rho /(R \theta)^{1 / 2}\right)
$$

and, hence, the rod behaves like a perfect gas under severe compression. This is not as unrealistic an assumption as it might appear to be, for it entails no restriction upon the behaviour of the rod under extension.

The following notation is adopted in what follows:

$$
\begin{aligned}
\phi(t) & =T(\theta(t)), \\
\psi(x, t) & =\hat{\psi}(\rho(x, t), \theta(t)), \\
\Psi(t) & =\int_{0}^{1} \psi d x, \\
K(t) & =\frac{1}{2} \int_{0}^{1} u_{t}^{2} d x, \\
E & =\Psi+K+p L .
\end{aligned}
$$

It will be shown that

$$
I(t)=\frac{E(t)}{\phi(t)^{2}}+3 p \int_{0}^{t} \frac{L(s) \dot{\phi}(s)}{\phi(s)^{3}} d s
$$

is an adiabatic invariant in certain circumstances.

Although $I$ is constructed from $E$, which is an energy, from the length $L$, the pressure $p$, and the environmental temperature $\theta$ (through $\phi$ ), the situation is somewhat different from Lorentz's problem in that the length is not prescribed at the outset.

A difficulty we shall have to bear in mind is that, whereas $\phi, K$, and $L$ are always positive, $\Psi$ need not be so. 
Slowly-varying temperatures. In order that $I$ be an adiabatic invariant, it is necessary to suppose the environmental temperature $\theta(t)$ to be slowly-varying in the sense that $\phi(t)=T(\theta(t))$ meets the hypotheses:

(i) $\phi(t)$ is $C^{2}$;

(ii) as $t \rightarrow \infty, \phi(t)$ converges to a finite limit, $\phi(\infty)$ say, and $\dot{\phi}(t) \rightarrow 0$;

(iii) there are positive constants $\varepsilon, M, m, C_{1}, C_{2}, C_{3}$ such that

$$
\begin{gathered}
0<m \leq \phi(t) \leq M, \quad|\dot{\phi}(t)| \leq C_{1} \varepsilon, \\
\int_{0}^{\infty}|\dot{\phi}(t)| d t \leq C_{2}, \quad \int_{0}^{\infty}|\ddot{\phi}(t)| d t \leq C_{3} \varepsilon .
\end{gathered}
$$

The constants $M, m, C_{1}, C_{2}, C_{3}$ are to be thought of as being independent of $\varepsilon$, while $\varepsilon$ itself measures the slowness with which the temperature varies. The hypotheses are satisfied, in particular, if $\phi(t)=\Phi(\varepsilon t)$, where:

(iv) $\Phi(s)$ is $C^{2}$;

(v) as $s \rightarrow \infty, \Phi(s)$ converges to a finite limit and $\dot{\Phi}(s) \rightarrow 0$;

(vi) there are positive constants $M, m, C_{1}, C_{2}, C_{3}$ such that

$$
\begin{gathered}
0<m \leq \Phi(s) \leq M, \quad|\dot{\Phi}(s)| \leq C_{1}, \\
\int_{0}^{\infty}|\dot{\Phi}(s)| d s \leq C_{2}, \quad \int_{0}^{\infty}|\ddot{\Phi}(s)| d s \leq C_{3} .
\end{gathered}
$$

In the statement of our result, and at various points in the proof, it is necessary to introduce positive constants $C, C_{4}$, and $C_{5}$, which are independent of $\varepsilon$ and depend, at most, upon the constants $a, b, M, m, C_{1}, C_{2}, C_{3}$ and the initial value $E(0)$.

An adiabatic invariant. Our task is to show that if $p$ is sufficiently large then $I$ is an adiabatic invariant in the sense that, to within an error $O(\varepsilon), I$ is constant throughout the semi-infinite interval $t \geq 0$; this is accomplished by proving a little more.

Let $\theta(t)$ be slowly-varying, and let $p \geq 2 a M^{3}$. Then, as $t \rightarrow \infty, I(t)$ converges to a finite limit $I(\infty)$; moreover, there is a positive constant $C$ such that

$$
|I(t)-I(\infty)| \leq C \varepsilon \quad(t \geq 0) .
$$

In order to begin the proof we introduce the integral

$$
F(t)=\frac{1}{2} \int_{0}^{1}(x+u)^{2} d x
$$

and proceed to derive the identities

$$
\begin{aligned}
& \dot{E}=\frac{\dot{\phi}}{\phi}\left(2 \Psi+\int_{0}^{1} \frac{\sigma}{\rho} d x\right), \\
& \dot{I}=-\frac{\dot{\phi}}{\phi^{3}} \ddot{F} .
\end{aligned}
$$

According to (3) and (4), $E$ and $I$ would be exactly constant if $\theta$, and hence $\phi$, were constant. 
To prove (3) we observe that

$$
\begin{aligned}
\dot{E} & =\dot{\Psi}+\dot{K}+p \dot{L} \\
& =\int_{0}^{1}\left(\psi_{t}+u_{t} u_{t t}\right) d x+p \dot{L} \\
& =\int_{0}^{1}\left(\hat{\psi}_{\theta} \dot{\theta}+\hat{\psi}_{\rho} \rho_{t}+\sigma_{x} u_{t}\right) d x+p \dot{L} \\
& =\dot{\theta} \int_{0}^{1} \hat{\psi}_{\theta} d x+\int_{0}^{1}\left(-\frac{\sigma}{\rho^{2}} \rho_{t}+\sigma_{x} u_{t}\right) d x+p \dot{L} \\
& =\dot{\theta} \int_{0}^{1} \hat{\psi}_{\theta} d x+\int_{0}^{1}\left(\sigma u_{x t}+\sigma_{x} u_{t}\right) d x+p \dot{L} \\
& =\dot{\theta} \int_{0}^{1} \hat{\psi}_{\theta} d x+\left[\sigma u_{t}\right]_{0}^{1}+p \dot{L},
\end{aligned}
$$

and it follows with the aid of the boundary conditions that

$$
\dot{E}=\dot{\theta} \int_{0}^{1} \hat{\psi}_{\theta} d x
$$

According to the identity (1),

$$
\dot{\theta} \int_{0}^{1} \hat{\psi}_{\theta} d x=\frac{T^{\prime}(\theta) \dot{\theta}}{T(\theta)}\left(2 \Psi+\int_{0}^{1} \frac{\sigma}{\rho} d x\right)=\frac{\dot{\phi}}{\phi}\left(2 \Psi+\int_{0}^{1} \frac{\sigma}{\rho} d x\right)
$$

and, hence, $(3)$ is correct.

To prove (4) we differentiate $F$ twice with respect to $t$ to find that

$$
\begin{aligned}
\ddot{F} & =\int_{0}^{1} u_{t}^{2} d x+\int_{0}^{1}(x+u) u_{t t} d x \\
& =2 K+\int_{0}^{1}(x+u) \sigma_{x} d x \\
& =2 K+[\sigma(x+u)]_{0}^{1}-\int_{0}^{1} \sigma\left(1+u_{x}\right) d x \\
& =2 K-p L-\int_{0}^{1} \frac{\sigma}{\rho} d x
\end{aligned}
$$

or, on substituting $E-\Psi-p L$ for $K$, that

$$
\ddot{F}=2 E-3 p L-2 \Psi-\int_{0}^{1} \frac{\sigma}{\rho} d x .
$$

On combining this last equation with (3), we obtain

$$
\dot{E}=\frac{\dot{\phi}}{\phi}(2 E-3 p L-\ddot{F}) \text {. }
$$

On the other hand, the definition (2) implies that

$$
\dot{I}=\frac{\dot{E}}{\phi^{2}}-\frac{2 E}{\phi^{3}} \dot{\phi}+\frac{3 p L}{\phi^{3}} \dot{\phi}
$$


and therefore (4) is correct.

Up to this point the hypothesis on $p$ has played no part; it is needed now, though, to ensure the validity of the estimates:

$$
\begin{aligned}
|\Psi| & \leq E, \\
K+p L & \leq 2 E, \\
|\dot{F}| & \leq 2 E^{3 / 2} /\left(a M^{3}\right) .
\end{aligned}
$$

According to (6), $E$ must be strictly positive whenever $p \geq 2 a M^{3}$.

To prove (5) we start from the hypothesis $\tau S(\tau) \geq-a$, which tells us that

$$
S\left(\frac{\rho}{T(\theta)}\right) \geq-a \frac{T(\theta)}{\rho}=-a T(\theta)\left(1+u_{x}\right)
$$

and, therefore,

$$
\int_{0}^{1} S\left(\frac{\rho}{T(\theta)}\right) d x \geq-a T(\theta)(1+u(1, t))=-a T(\theta) L .
$$

Hence,

$$
\Psi=T(\theta)^{2} \int_{0}^{1} S\left(\frac{\rho}{T(\theta)}\right) d x \geq-a T(\theta)^{3} L=-a \phi^{3} L,
$$

and because $0<\phi \leq M$ we have

$$
\Psi \geq-a M^{3} L
$$

Now (5) is certainly correct at any instant $t$ at which $\Psi(t) \geq 0$, for then

$$
E(t)=\Psi(t)+K(t)+p L(t) \geq \Psi(t)=|\Psi(t)| .
$$

On the other hand, if $\Psi(t)<0$ and $p \geq 2 a M^{3}$ we can invoke (8) to deduce that

$$
\begin{aligned}
E(t) & =\Psi(t)+K(t)+p L(t) \\
& \geq \Psi(t)+K(t)+2 a M^{3} L(t) \\
& \geq \Psi(t)+K(t)-2 \Psi(t) \\
& =K(t)-\Psi(t) \\
& =K(t)+|\Psi(t)| \geq|\Psi(t)|
\end{aligned}
$$

and so (5) is always correct.

Since

$$
K+p L \leq K+p L+\Psi+|\Psi|=E+|\Psi| \leq 2 E,
$$

(6) is an immediate deduction from (5).

To prove (7) we start from the equation

$$
\dot{F}=\int_{0}^{1}(x+u) u_{t} d x
$$

and apply Schwarz's inequality to obtain the estimate

$$
\dot{F}^{2} \leq \int_{0}^{1}(x+u)^{2} d x \int_{0}^{1} u_{t}^{2} d x=2 K \int_{0}^{1}(x+u)^{2} d x .
$$


As has been remarked already, $0 \leq x+u \leq L$ and so

$$
\dot{F}^{2} \leq 2 K L^{2}=2 K(p L)^{2} / p^{2},
$$

and on appealing to $(6)$ we see that

$$
\dot{F}^{2} \leq 16 E^{3} / p^{2} \leq 4 E^{3} /\left(a M^{3}\right)^{2},
$$

which is (7).

The next step in the argument is to return to the identity (3) and use it to establish that $E(t)$ converges to a limit as $t \rightarrow \infty$; this part of the proof depends upon the hypothesis $\tau\left|S^{\prime}(\tau)\right| \leq b$. We have

$$
\frac{\sigma}{\rho}=-\rho T(\theta) S^{\prime}\left(\frac{\rho}{T(\theta)}\right)=-T(\theta)^{2} \frac{\rho}{T(\theta)} S^{\prime}\left(\frac{\rho}{T(\theta)}\right)
$$

and, therefore,

$$
\frac{|\sigma|}{\rho} \leq b T(\theta)^{2}=b \phi^{2} \leq b M^{2} .
$$

When we combine this last estimate with (3) and (5) we see that

$$
|\dot{E}| \leq \frac{|\dot{\phi}|}{\phi}\left(2|\Psi|+\int_{0}^{1} \frac{|\sigma|}{\rho} d x\right) \leq \frac{|\dot{\phi}|}{m}\left(2 E+b M^{2}\right) .
$$

Thus the integral

$$
\int_{0}^{\infty}\left|\frac{\cdot}{\log \left(E+\frac{1}{2} b M^{2}\right)}\right| d t
$$

must converge because it equals

$$
\int_{0}^{\infty} \frac{|\dot{E}|}{E+\frac{1}{2} b M^{2}} d t \leq \frac{2}{m} \int_{0}^{\infty}|\dot{\phi}| d t \leq \frac{2 C_{2}}{m} .
$$

The convergence of the integral ensures that $\log \left(E(t)+\frac{1}{2} b M^{2}\right)$ must converge to a limit as $t \rightarrow \infty$ and, therefore, $E(t)$ itself must converge to a limit.

In fact, we can say a little more, for

$$
\begin{aligned}
\log \left(\frac{E(t)+\frac{1}{2} b M^{2}}{E(0)+\frac{1}{2} b M^{2}}\right) & =\int_{0}^{t} \frac{.}{\log \left(E(s)+\frac{1}{2} b M^{2}\right)} d s \\
& \leq \int_{0}^{\infty}\left|\overline{\log \left(E(s)+\frac{1}{2} b M^{2}\right)}\right| d s \leq \frac{2 C_{2}}{m}
\end{aligned}
$$

and, therefore,

$$
E(t) \leq E(t)+\frac{1}{2} b M^{2} \leq\left(E(0)+\frac{1}{2} b M^{2}\right) \exp \left(2 C_{2} / m\right)=C_{4},
$$

where $C_{4}$ is independent of $\varepsilon$.

Next, we observe that, as (6) tells us, $0<p L \leq 2 E \leq 2 C_{4}$. Thus

$$
\frac{p L|\dot{\phi}|}{\phi^{3}} \leq \frac{2 C_{4}}{m^{3}}|\dot{\phi}|
$$


and, because

$$
\int_{0}^{\infty}|\dot{\phi}| d t \leq C_{2}
$$

the integral

$$
\int_{0}^{\infty} \frac{L(s) \dot{\phi}(s)}{\phi(s)^{3}} d s
$$

must converge. On returning to the definition (2) and remembering that $E(t)$ and $\phi(t)$ converge to limits as $t \rightarrow \infty$, we see that the adiabatic invariant $I(t)$ must converge to a limit $I(\infty)$.

It remains to check that $I=I(\infty)+O(\varepsilon)$. To do so we integrate (4) by parts to deduce that, for any $t \geq 0$ and any $t_{1}>t$,

$$
\begin{aligned}
I\left(t_{1}\right)-I(t) & =-\int_{t}^{t_{1}} \frac{\dot{\phi}(s)}{\phi(s)^{3}} \ddot{F}(s) d s \\
& =-\left[\frac{\dot{\phi} \dot{F}}{\phi^{3}}\right]_{t}^{t_{1}}+\int_{t}^{t_{1}}\left(\frac{\ddot{\phi}(s)}{\phi(s)^{3}}-\frac{3 \dot{\phi}(s)^{2}}{\phi(s)^{4}}\right) \dot{F}(s) d s .
\end{aligned}
$$

According to (7), $|\dot{F}| \leq 2 C_{4}^{3 / 2} /\left(a M^{3}\right)=C_{5}$ and, therefore,

$$
\begin{aligned}
\left|\frac{\ddot{\phi}}{\phi^{3}}-\frac{3 \dot{\phi}^{2}}{\phi^{4}}\right||\dot{F}| & \leq \frac{C_{5}}{m^{3}}|\ddot{\phi}|+\frac{3 C_{5}|\dot{\phi}|^{2}}{m^{4}} \\
& \leq \frac{C_{5}}{m^{3}}|\ddot{\phi}|+\frac{3 C_{1} C_{5} \varepsilon}{m^{4}}|\dot{\phi}|,
\end{aligned}
$$

where, by hypothesis, $\dot{\phi}$ and $\ddot{\phi}$ are integrable on $[0, \infty)$. Thus if we let $t_{1} \rightarrow \infty$ in (9) and remember that $I\left(t_{1}\right) \rightarrow I(\infty), \dot{\phi}\left(t_{1}\right) \rightarrow 0$, and $\left|\dot{F}\left(t_{1}\right)\right| \leq C_{5}$, we see that

$$
I(\infty)-I(t)=\frac{\dot{\phi}(t) \dot{F}(t)}{\phi(t)^{3}}+\int_{t}^{\infty}\left(\frac{\ddot{\phi}(s)}{\phi(s)^{3}}-\frac{3 \dot{\phi}(s)^{2}}{\phi(s)^{4}}\right) \dot{F}(s) d s,
$$

the convergence of the integral being assured.

Finally, we estimate the right-hand side of (10) to find, as required, that

$$
|I(\infty)-I(t)| \leq \frac{C_{1} C_{5} \varepsilon}{m^{3}}+\frac{C_{3} C_{5} \varepsilon}{m^{3}}+\frac{3 C_{1} C_{2} C_{5} \varepsilon}{m^{4}}=C \varepsilon
$$

\section{REFERENCES}

[1] W. A. Day, Adiabatic invariants for strings and membranes subjected to slowly-varying tension, Quart. Appl. Math. 45, 349-360 (1987)

[2] W. A. Day, Adiabatic invariants for strings, membranes, and beams of slowly-varying dimensions, Quart. Appl. Math. 45, 389-398 (1987)

[3] J. E. Littlewood, Lorentz's pendulum problem, Ann. Phys. 21, 233-242 (1963)

[4] Collected papers of J. E. Littlewood, Vol. I, edited by a committee appointed by the London Mathematical Society, Clarendon Press, Oxford, 1982 